\title{
Chemical Processing of an Early Salt Print Photograph Revealed with X-ray Fluorescence Elemental Mapping
}

\author{
Daryl L. Howard ${ }^{1, *}$, Martin D. de Jonge ${ }^{1}$ and David Paterson ${ }^{1}$ and David Thurrowgood ${ }^{2}$ \\ 1. Australian Synchrotron (ANSTO), Clayton, Australia. \\ 2. Queen Victoria Museum and Art Gallery, Launceston, Australia. \\ * Corresponding author, daryl.howard@synchrotron.org.au
}

The salt print was an early paper-based photographic process for producing positive prints in the mid$19^{\text {th }}$ century. We have analysed a salt print with high-definition fast-scanning X-ray fluorescence (XRF) microscopy, which can non-destructively reveal elemental distributions, and hence learn about the chemical processes used in the print's creation. To date, the majority of elemental studies regarding historic photographs have relied on XRF point analysis, in which a few points of interest are analysed at low resolution [1].

The salt print photograph (Fig. 1a) was scanned at the XFM beamline with a Kirkpatrick-Baez microprobe using the Maia detector array with an incident energy $18.5 \mathrm{keV}, 15$ micron pixel size, $5 \mathrm{~ms}$ dwell time, and $3 \mathrm{~mm} / \mathrm{s}$ scan velocity for overall scan time of $17.8 \mathrm{~h} \mathrm{[2]}$. The event-mode data was processed into 12.8 Mpixel elemental concentration maps with GeoPIXE software [3].

Silver is a key element in the elemental analysis of historic photographs. We had to rely on Ag-L fluorescence for its detection. The relatively low energy $\mathrm{Ag}-\mathrm{L}$ fluorescence $\left(\mathrm{L} \alpha_{1}=2.98 \mathrm{keV}\right)$ is a challenge to detect in air, partly because of its moderate attenuation (transmission $=82 \%$ in $10 \mathrm{~mm}$ air), but primarily because it overlaps with atmospheric Ar-K fluorescence $\left(\mathrm{K}_{1}=2.96 \mathrm{keV}\right)$. Argon constitutes $0.934 \%$ by volume in air, so its fluorescence intensity can dominate that of trace elements of similar fluorescence energy. Optimal sensitivity to Ag-L fluorescence would be achieved in a vacuum environment, but such a setup is not practical at the XFM beamline. To help reduce argon from the sample environment, a polycarbonate enclosure with a Kapton window was mounted to the Maia detector, to which was delivered a continuous flow of nitrogen $(\sim 0.9 \mathrm{~L} / \mathrm{min})$. The bottom of the enclosure is partially open to allow sample access and permit raster scanning.

The silver distribution map (Fig. 1b) reveals the portrait as would be expected from a salt print. The gold map (Fig. 1c) yields the clearest image of the original photograph of all the elemental maps observed. This is partly due to the greater sensitivity to Au-L detection versus Ag-L at the XFM beamline. Gold was used to tone photographs and to improve image stability [4].

Mercury (Fig. 1d) is an interesting find, as it is typically considered to be absent from salt prints [1]. Mercuric iodide or mercuric chloride was sometimes used by photographers to chemically intensify the original silver image via the addition of optically denser mercury to silver [4]. The mottled appearance of mercury warrants further investigation, as the $\mathrm{Hg}$ spots do not collocate with other elements observed.

The halogens chlorine and bromine (Fig. 1e and 1f) are common in the many salts used in photographic processes, and both are observed to slightly contribute to the image chemistry. Iodine was observed in several spots of approximately $0.3 \mathrm{~mm}$ diameter, which appear to be part of the paper support rather than the photographic image and is not presented. 
Lead was present on the photograph (Fig. 1g), but does not appear to be part of the photographic processing. Interestingly, fingerprints containing $\mathrm{Pb}$ were observed on the right side, below centre of the print. This observation would not be possible with conventional XRF point analysis. The source of lead is unknown, but this finding is evidence to its widespread use in the past.

The dotted copper distribution (Fig. 1h) appears to be part of the paper support. Zinc and trace nickel are highly collocated with copper and are not shown. The $\mathrm{Cu}: \mathrm{Zn}$ concentration ratio varies from spot to spot, but is generally $2: 1$. This suggests brass inclusions are present in the paper. Metal inclusions in paper can result from particles abraded from the metal equipment or and/or contaminated water used in the papermaking process [5].

These findings show that scanning XRF is a powerful tool for the determination of chemical processing techniques of historic photographs. The spatially resolved elemental distribution maps maintain the context of the photographic image, thus determination of the source of the elemental signal is greatly simplified [6].
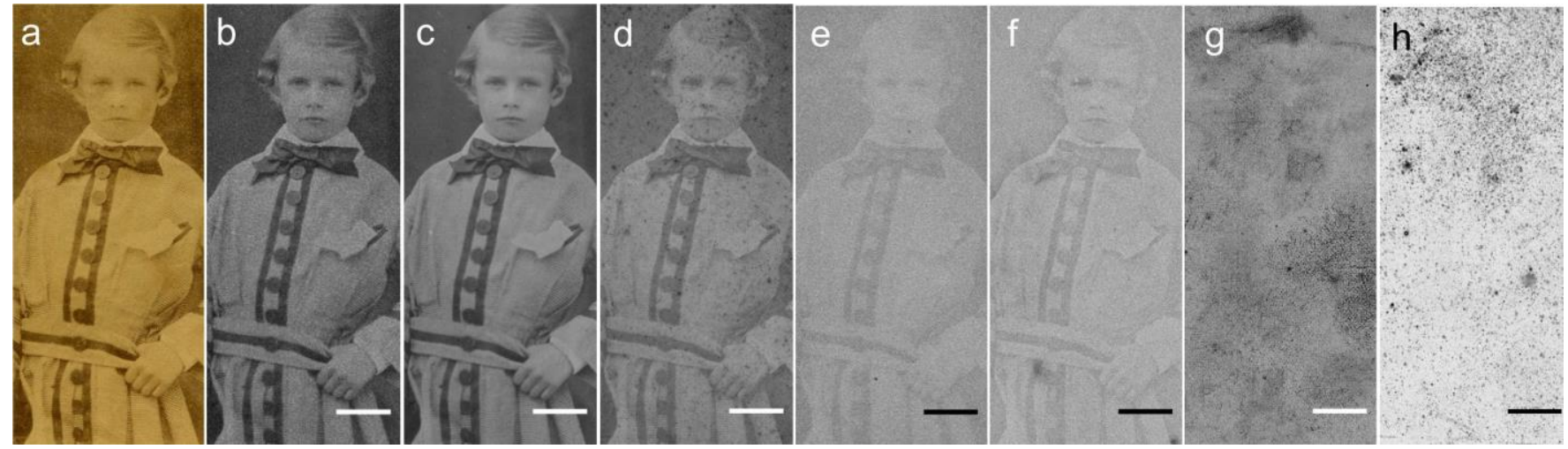

Figure 1. a) Visible light image of a salt print photograph, circa 1850, photographer unknown. Scanning XRF elemental maps of: b) silver, c) gold, d) mercury, e) chlorine, f) bromine, g) lead and h) copper. Scale bar $=10 \mathrm{~mm}$. Darker areas indicate higher concentrations.

\section{References}

[1] DC Stulik and A Kaplan "Salt Print" (Getty Conservation Institute, Los Angeles, 2013)

[2] D Paterson et al, AIP Conf. Proc. 1365 (2011) 219-222.

[3] CG Ryan et al, Nucl. Instrum. Methods Phys. Res., B. 47 (1990) 55-71.

[4] CEK Mees "Theory of the Photographic Process" (Macmillan, New York, 1942).

[5] S Choi, J. Am. Inst. Cons. 46 (2007) 137-152.

[6] This research was undertaken at the XFM beamline at the Australian Synchrotron, part of ANSTO. 